\title{
Solution of pressure gradient stretching plate with suction
}

\author{
Ramesh B. Kudenatti ${ }^{\mathrm{a}, *}$, Vishwanath B. Awati ${ }^{\mathrm{b}}$ \\ a Department of Mathematics, Bangalore University, Bangalore 560 001, India \\ ${ }^{\mathrm{b}}$ Department of Mathematics, Government First Grade College, Kunigal 572 130, India
}

\section{A R T I C L E I N F O}

\section{Keywords:}

Falkner-Skan equation

Similarity transformation

Power series

Stretching of variables

Least squares approximation

\begin{abstract}
A B S T R A C T
Solutions for the boundary value problem over an infinite domain have been obtained by first transforming the two-dimensional laminar boundary layer equations into an ordinary differential equation through similarity variables. The governing problem is the twoparameter Falkner-Skan equation with $\beta$, the streamwise pressure gradient and $\gamma$ the suction velocity. The closed form solution for $\beta=-1$ obtained earlier is rewritten, which is then generalized for general $\beta$. The same equation is also solved using method of stretching of variables. Different velocity profiles have been observed for both $\beta$ and $\gamma$. The results from both approaches are compared with that of direct numerical solutions, which agree very well.
\end{abstract}

(c) 2008 Elsevier Inc. All rights reserved.

\section{Introduction}

Boundary layer flows on continuous stretching surfaces moving in quiescent fluid medium in industrial manufacturing processes are of present interest in recent years. One can assume that stretching of the boundary varies with distance from the die in the manner of power law. For example, flows along the liquid films in condensation processes, cooling of a metallic plate in a cooling bath, aerodynamic extrusion of plastic sheets, drawing of plastic films and metal and polymer extrusion, etc. Both stretching and heating or cooling simultaneously during such processes has a significant effect on the final products.

Boundary value problems over an infinite interval are of special interest and a very few of them have got analytical solutions. The continuous stretching surface is governed by these BVP's, which are theoretically studied with the aid of similarity form of the two dimensional boundary layer equations. Earliest study is by Sakiadis [1] who investigated the boundary layer flow on a continuous solid surface with constant speeds. Afzal and Varshney [2], Afzal [3,4] have investigated the self-similar boundary layer with power law stretching of the continuous surface. The comprehensive analytical and numerical study of boundary layer flows induced by continuous surface stretched with power law velocity has been given in Banks [5]. The stretching surface may be considered either as an impermeable or permeable one.

The pressure gradient driven flow over a stretching boundary may be obtained when external and the boundary velocity are proportional to the power law of the downstream coordinate and is governed by the Falkner-Skan equation. Weyl [6] proved the existence and uniqueness of the solution of the Falkner-Skan equation for impermeable surface. Coppel [7] proved existence and uniqueness of the solution of the Falkner-Skan equation. Particularly, he proved that for non-negative $f(0)$ and $f(0), f^{\prime}(\eta)$ is positive, zero or negative throughout the interval $0 \leqslant \eta \leqslant \infty$ according to $f(0)$ is less than, equal or greater than one and with this restriction on $f^{\prime \prime}(\eta)$, the solution is unique. Yang and Chien [8] give two types of analytical solutions of the Falkner-Skan equation for $\beta=-1$, one expressed in terms of error and exponential functions and other one in the form of confluent hypergeometric functions. Riley and Weidman [9] give numerically a rich

\footnotetext{
* Corresponding author.

E-mail address: ramesh@bub.ernet.in (R.B. Kudenatti).
} 
structure of solution for this equation for $|\beta| \leqslant 1$ and show no solution, single, double and triple solutions for particular values of $\beta$. Afzal [10] recently formulated another version of the Falkner-Skan equation, which relates free stream velocity to composite reference velocity (i.e. sum of the velocities of stretching boundary and free stream). He also gave closed form solution for $\beta=-1$ and asymptotic solutions for some special cases. Recently, Sachdev et al. [11] have proposed a new scheme for the solution of the Falkner-Skan equation for general $\beta$ and recovered all possible results of Riley and Weidman [9].

In this paper, an attempt has been made to give exact solution of the Falkner-Skan equation for general $\beta$ and for different values of suction parameter $\gamma$. The presentation of the paper proceeds as follows. In Section 2, the mathematical formulation of the proposed problem with relevant boundary conditions is given. The exact analytical solution for $\beta=-1$ as obtained by Yang and Chien [8] can be rewritten in the form $f=\eta+\delta+\frac{\gamma-\delta}{G(\eta)}$ and the function $G(\eta)$ is defined by infinite power series. This form is used for the solution of the Falkner-Skan equation for general $\beta$ which is the main objective of the present work. This analysis is given in Section 3. In Section 4, the solution of the Falkner-Skan equation by means of the method of stretching of variables is given. Section 5 is devoted to compare the results obtained in Sections 3 and 4.

\section{Formulation of the problem}

The two-dimensional laminar boundary layer equations of an incompressible fluid subjected to pressure gradient are

$$
\begin{aligned}
& \frac{\partial u}{\partial x}+\frac{\partial v}{\partial y}=0, \\
& u \frac{\partial u}{\partial x}+v \frac{\partial u}{\partial y}=-\frac{p^{\prime}}{\rho}+v \frac{\partial^{2} u}{\partial y^{2}},
\end{aligned}
$$

where $p^{\prime}$ is pressure gradient, $p^{\prime}=-\rho U \frac{d U}{d x}, u$ is the velocity in the direction of the fluid, $v$ is the velocity of the fluid normal to $u, v$ is the constant kinematic viscosity, $U$ is the velocity at the edge of boundary layer which obeys the power law relation $U(x)=U_{\infty} x^{m}$. The relevant boundary conditions are

$$
\text { at } y=0: u=0, \quad v=v_{w}, \quad \text { and at } y \rightarrow \infty: u=U_{\infty} .
$$

The stream functions $u=\frac{\partial \psi}{\partial y}$ and $v=-\frac{\partial \psi}{\partial x}$, satisfy Eq. (1) where

$$
\psi=\sqrt{\frac{2 v x U(x)}{1+m}} f(\eta), \quad \eta=y \sqrt{\frac{(1+m) U(x)}{2 v x}}, \quad \beta=\frac{2 m}{1+m}
$$

and $f$ is the dimensionless stream function. Using these similarity transformations in Eqs. (1)-(3), we get a nonlinear ordinary differential equation

$$
f^{\prime \prime \prime}+f f^{\prime \prime}+\beta\left(1-f^{\prime 2}\right)=0, \quad '=\frac{d}{d \eta}
$$

with boundary conditions

$$
f(0)=\gamma, \quad f^{\prime}(0)=0 \quad \text { and } f^{\prime}(\infty)=1,
$$

where $\gamma$ corresponds to the suction velocity, considered mainly positive in the present study and $\gamma=0$ corresponds to an impermeable surface. Eq. (5) is the Falkner-Skan equation, one of the celebrated equations in the similarity boundary layer theory and governs the symmetrical boundary layer flow over a wedge whose included angle is $\pi \beta$. In the forthcoming section, the exact solution for the system (5) and (6) for general $\beta$ which embeds the known analytical solution for $\beta=-1$, is given.

\section{Solution for general $\beta$}

The exact analytical solution of the Falkner-Skan equation (5) subjected to (6) for $\beta=-1$ as given by Yang and Chien [8] is rewritten in slightly modified form as

$$
f=\eta+\delta+\frac{\gamma-\delta}{G(\eta)}
$$

where

$$
G(\eta)=e^{\frac{\eta^{2}}{2}+\eta \delta}-\frac{1}{2} \sqrt{\frac{\pi}{2}}(\gamma-\delta) e^{\frac{1}{2}(\eta+\delta)^{2}}\left(\operatorname{erf}\left(\frac{\delta}{\sqrt{2}}\right)-\operatorname{erf}\left(\frac{\eta+\delta}{\sqrt{2}}\right)\right)
$$

and $\delta=\sqrt{\gamma^{2}-2}$. The axial velocity gradient at the wall is

$$
f^{\prime \prime}(0)=\delta= \pm \sqrt{\gamma^{2}-2} .
$$


From Eq. (9) for $\beta=-1$, the solution exists for $\gamma>\sqrt{2}$ and no solution for $\gamma<\sqrt{2}$. Indeed we can find solution of system (5), (6) for $\gamma<\sqrt{2}$ through the method of stretching of variables. The function $G$ in Eq. (7) easily generalizes the solution of the Falkner-Skan equation for general $\beta$. For general $\beta$, in view of the transformation (7), Eq. (5) becomes

$$
G^{2} G^{\prime \prime \prime}-G\left[6 G^{\prime}+(\delta-\gamma)-(\eta+\delta) G\right] G^{\prime \prime}+6 G^{\prime 3}+[(\gamma-\delta)(\beta-2)-2(\eta+\delta) G] G^{2}-2 \beta G^{2} G^{\prime}=0
$$

and the boundary conditions (6) become

$$
G(0)=1, \quad G^{\prime}(0)=\frac{1}{\gamma-\delta}, \quad G(\infty)=\infty .
$$

The solution of (10) subjected to the conditions (11) for $\beta=-1$ is given by Eq. (8) which is expressed in terms of exponential and error functions. These functions may be expanded in Taylor series about $\eta=0$, which enjoy the infinite radius of convergence. The solution of the problem (10) for $\beta=-1$ gives a clue for the following analysis for general $\beta$. Motivated by the series representation of $G(\eta)$ for $\beta=-1$, it is natural to write

$$
G=\sum_{n=0}^{\infty} a_{n} \eta^{n}
$$

for general $\beta$. Substitution of (12) with $a_{0}=1, a_{1}=1 /(\gamma-\delta)$ into (10) and after equating the like powers of $\eta$, the general recurrence relation for obtaining the coefficients $a_{n}$, we get

$$
\begin{aligned}
a_{n+3}= & -\frac{1}{(1+n)(2+n)(3+n)}\left[\sum_{m=0}^{n-1} \sum_{i=0}^{n-m}(1+m)(2+m)(3+m) a_{n-m-i} a_{i} a_{m+3}+\sum_{m=0}^{n}\left\{-(\delta-\gamma)(1+m)(2+m) a_{n-m} a_{m+2}\right.\right. \\
& +(\gamma-\delta)(\beta-2)(n-m+1)(1+m) a_{n-m+1} a_{m+1}+\sum_{i=0}^{n-m}\left\{m(1+m) a_{n-m-i} a_{i} a_{m+1}+\delta(1+m)\right. \\
& \times(2+m) a_{n-m-i} a_{i} a_{m+2}-2 \beta(1+m) a_{m+1} a_{i} a_{n-m-i}-6(1+i)(1+m)(2+m) a_{n-m-i} a_{i+1} a_{m+2} \\
& \left.-2(n-m-i)(1+i) a_{n-m-i} a_{i} a_{m}\right\}+u m_{i=0}^{m}\left\{-2 \delta(1+i)(m-i+1) a_{n-m} a_{i+1} a_{m-i+1}\right. \\
& \left.\left.\left.+6(n-m+1)(m-i+1)(i+1) a_{n-m+1} a_{m-i+1} a_{i+1}\right\}\right\}\right] \quad \text { for } n=0,1,2,3, \ldots
\end{aligned}
$$

All the coefficients $a_{n}$ in (13) are obtained in terms of $a_{2}$ and it is related to $f^{\prime}(0)$ as

$$
a_{2}=-\left(f^{\prime \prime}(0)-\frac{2}{\gamma-\delta}\right) /(2(\gamma-\delta)) .
$$

The series solution (12) as it is contains two unknown constants namely $\delta$ and $f^{\prime}(0)$ or $a_{2}$. Thus, we have a two-parameter family of solutions to the system (5), (6). To determine one of these unknown constants, we patch the series expansion of (8) with that of (12), which gives $\delta=\sqrt{\gamma^{2}-2}$. Another free parameter $f^{\prime}(0)$ or $a_{2}$ gives one parameter family of solutions to the system (5) and (6). This constant can be found from the following integral relation that is obtained by integrating the system (5) and (6)

$$
\int_{0}^{\infty}\left(f^{\prime}-f^{\prime 2}\right) d \eta+\beta \int_{0}^{\infty}\left(1-f^{\prime^{2}}\right) d \eta=f^{\prime \prime}(0)-\gamma .
$$

\begin{tabular}{|c|c|c|c|c|c|c|c|c|}
\hline \multirow[t]{2}{*}{$\beta$} & \multicolumn{2}{|l|}{$\gamma=1.5$} & \multicolumn{2}{|l|}{$\underline{\gamma}=2.0$} & \multicolumn{2}{|l|}{$\gamma=2.5$} & \multicolumn{2}{|l|}{$\underline{\gamma}=3.0$} \\
\hline & Eq. (7) & Numerical solution & Eq. (7) & Numerical solution & Eq. (7) & Numerical solution & Eq. (7) & Numerical solution \\
\hline-1 & 0.5 & 0.5 & 1.41421 & 1.41423 & 2.06155 & 2.06160 & 2.64575 & 2.64579 \\
\hline 0.0 & 1.72129 & 1.73192 & 2.19328 & 2.19453 & 2.65917 & 2.66657 & 3.14166 & 3.14511 \\
\hline 0.5 & 2.02156 & 2.02617 & 2.46585 & 2.45100 & 2.88354 & 2.89237 & 3.34547 & 3.34583 \\
\hline 1.0 & 2.26751 & 2.26761 & 2.66874 & 2.67002 & 3.09451 & 3.09112 & 3.52539 & 3.52663 \\
\hline 1.5 & 2.47156 & 2.47660 & 2.86367 & 2.86392 & 3.27094 & 3.27042 & 3.69299 & 3.69234 \\
\hline
\end{tabular}

Since, function $f$ in right hand side also involves $f^{\prime}(0)$, we need to find it iteratively for different values of $\beta$ and $\gamma$. The value $f^{\prime}(0)$ thus obtained agrees well with that of numerical solution and is given in the Table 1 for various values of $\beta$ and $\gamma$. The solutions for velocity profile $f$ for different values of stretching parameter $\beta$ and for suction parameter $\gamma \geqslant \sqrt{2}$ are given in Fig. 1a-d.

The relations ( 7 ) and (12) give the solution for general $\beta$, but restricts the analysis for $\gamma \geqslant \sqrt{2}$. In the next section, we give the solution for general $\beta$ and $\gamma$ by means of method of stretching of variables.

Table 1

Comparison of $f^{\prime}(0)$ obtained from Eqs. (7) and (12) with that numerical solution for various $\beta$ and $\gamma$. 

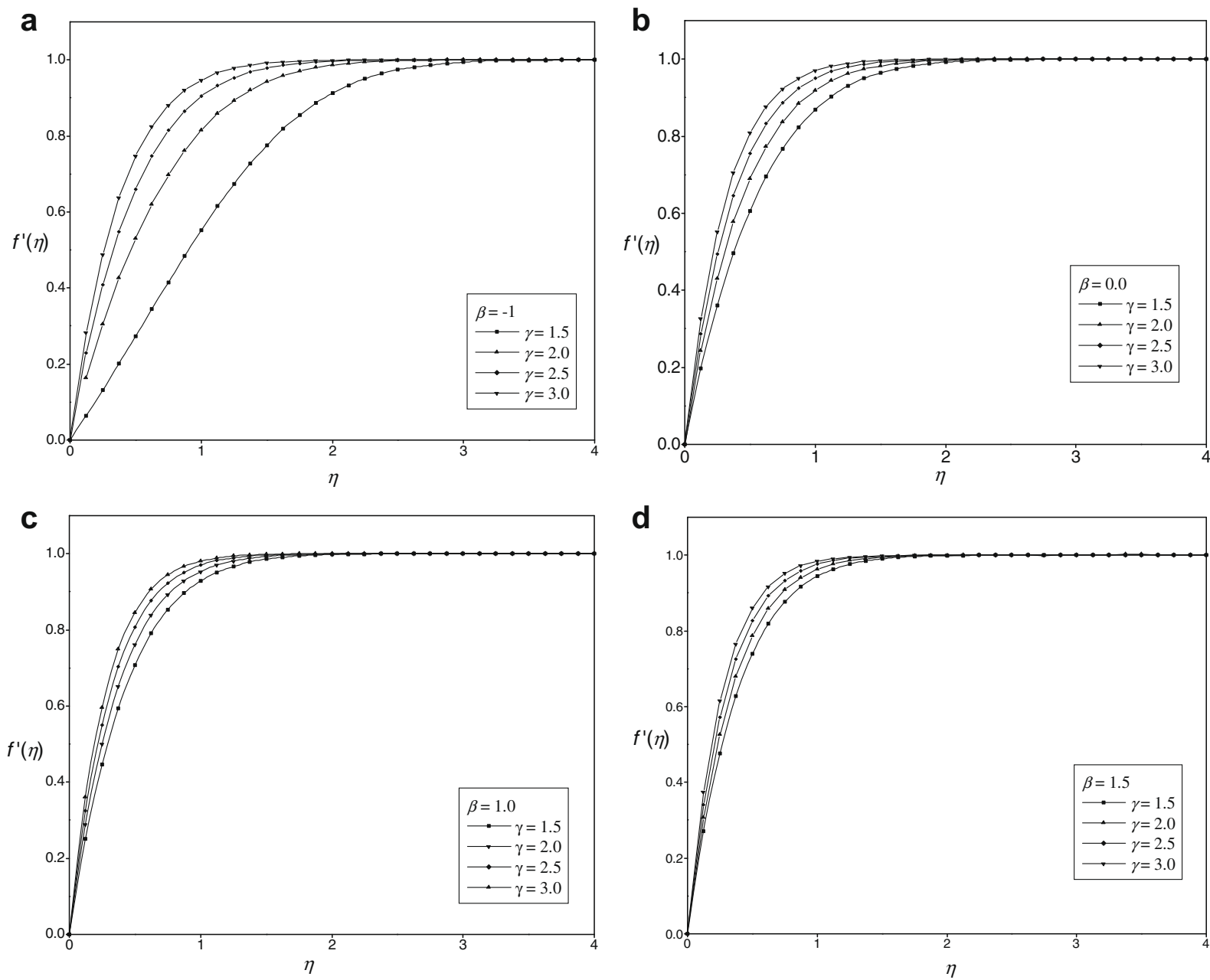

Fig. 1. Velocity profile $f(\eta)$ against $\eta$ for different values of stretching parameter $\beta$ and suction parameter $\gamma$.

\section{Method of stretching of variables}

Many attempts have been made to develop methods for the solution of boundary layer equations, which are approximate because these are not universally valid solutions especially for all possible distribution of the pressure gradient. The numerical approach is always based on the idea of stretching of variables of the flow problems. In the method of stretching of variables, we have to choose suitable derivative function $f$ such that the derivative boundary conditions satisfy automatically and integration of $f^{\prime}$ will satisfy the remaining boundary conditions. Substitution of this resulting function into the given equation gives the residual of the form $R(\xi, \alpha)$ and is called defect function. Using least square method, the residual of the defect function can be minimized. Afzal [12] has studied the least square method for minimization of the residual in the boundary layer equation. The solution to the third order nonlinear boundary value problem over an infinite domain characterizing the flow of a viscous fluid impinging normally to a wall from which the fluid is extracted at a uniform rate has been given by Ariel [13] using an method of stretching of variables. Chakraborty and Mazumdar [14] have given an approximate solution to the problem of steady laminar flow of MHD fluid over a stretching sheet. More details about the method of stretching of variables are given in [13,14].

Using the transformation $f=\gamma+F$, the system (5) and (6) reads as

$$
\begin{aligned}
& F^{\prime \prime \prime}+(\gamma+F) F^{\prime \prime}+\beta\left(1-F^{2}\right)=0, \quad '=\frac{d}{d \eta}, \\
& F(0)=0, \quad F^{\prime}(0)=0, \quad F^{\prime}(\infty)=1 .
\end{aligned}
$$

We introduce a stretching parameter $\alpha$ for both $F$ and $\eta$ in the form

$$
H(\xi)=\alpha F(\eta) \text { and } \xi=\alpha \eta,
$$


Table 2

Comparison of solutions (7) and (12) and solution (26) with numerical solution of the Falkner-Skan equations (5), (6)) with $\beta=2.0$ and $\gamma=1.5$.

\begin{tabular}{|c|c|c|c|}
\hline$\eta$ & $\begin{array}{l}\text { Eqs. (7) and (12) } \\
f(\eta)\end{array}$ & $\begin{array}{l}\text { Eq. (26) } \\
f^{\prime}(\eta)\end{array}$ & $\begin{array}{l}\text { Numerical solution } \\
f^{\prime}(\eta)\end{array}$ \\
\hline 0.0 & 0.0 & 0.0 & 0.0 \\
\hline 0.125 & 0.2890527 & 0.27682 & 0.2890416 \\
\hline 0.250 & 0.5022514 & 0.477011 & 0.5022322 \\
\hline 0.375 & 0.6563715 & 0.621785 & 0.6563484 \\
\hline 0.5 & 0.7658656 & 0.726482 & 0.7658432 \\
\hline 0.625 & 0.8424585 & 0.802198 & 0.8424404 \\
\hline 0.750 & 0.8952774 & 0.856953 & 0.8952665 \\
\hline 0.875 & 0.9312148 & 0.896551 & 0.9312129 \\
\hline 1.0 & 0.9553516 & 0.925188 & 0.9553597 \\
\hline 1.125 & 0.9713586 & 0.945898 & 0.9713774 \\
\hline 1.250 & 0.9998003 & 0.960874 & 0.9818719 \\
\hline 1.375 & 0.9886231 & 0.971705 & 0.9886639 \\
\hline 1.50 & 0.9929546 & 0.979538 & 0.9930068 \\
\hline 1.625 & 0.9956868 & 0.985202 & 0.9957507 \\
\hline 1.750 & 0.9973883 & 0.989298 & 0.9974644 \\
\hline 1.875 & 0.9984343 & 0.992261 & 0.9985232 \\
\hline 2.0 & 0.9990686 & 0.994403 & 0.9991712 \\
\hline 2.125 & 0.9994479 & 0.995952 & 0.9995653 \\
\hline 2.250 & 0.9996712 & 0.997073 & 0.9998045 \\
\hline 2.375 & 0.9998003 & 0.997883 & 0.9999508 \\
\hline 2.50 & 0.9999154 & 0.998469 & 1.0000425 \\
\hline 2.625 & 0.9999630 & 0.998893 & 1.0001028 \\
\hline 2.750 & 0.9999935 & 0.999199 & 1.0001455 \\
\hline 2.875 & 1.0004284 & 0.999421 & 1.0001789 \\
\hline 3.0 & 1.0004584 & 0.999581 & 1.0002079 \\
\hline 3.125 & 1.0008525 & 0.999697 & 1.0002353 \\
\hline 3.250 & 1.0009666 & 0.999781 & 1.0002628 \\
\hline 3.375 & 1.0000199 & 0.999842 & 1.0002913 \\
\hline 3.50 & 1.0000349 & 0.999885 & 1.0003214 \\
\hline 3.625 & 1.0000560 & 0.999917 & 1.0003535 \\
\hline 3.750 & 1.0000723 & 0.99994 & 1.0003879 \\
\hline 3.875 & 1.0000872 & 0.999957 & 1.0004247 \\
\hline 4.0 & 1.0000978 & 0.999969 & 1.0004640 \\
\hline
\end{tabular}

where $\alpha>0$, is an amplification factor. In view of (18), the system (16) and (17) can be rewritten as

$$
\begin{aligned}
& \alpha^{2} H^{\prime \prime \prime}+\gamma \alpha H^{\prime \prime}+H H^{\prime \prime}+\beta\left(1-H^{\prime 2}\right)=0, \quad '=\frac{d}{d \xi}, \\
& H(0)=0, \quad H^{\prime}(0)=0, \quad H^{\prime}(\infty)=1 .
\end{aligned}
$$

We choose a trial function

$$
H^{\prime}=1-\exp (-\xi),
$$

which satisfy both derivative conditions in (20). Integrating (21) with respect to $\xi$ from 0 to $\xi$ using first condition of (20), we get

$$
H=\exp (-\xi)+\xi-1 .
$$

Substituting (22) into (19) we get the residual defect function $R(\xi, \alpha)$ as

$$
R(\xi, \alpha)=\left(-\alpha^{2}+\gamma \alpha+\xi+2 \beta-1\right) \exp (-\xi)+(1-\beta) \exp (-2 \xi) .
$$

Using the least square method as discussed in Ariel [13], for the minimization of Eq. (23), we have

$$
\frac{\partial}{\partial \alpha} \int_{0}^{\infty} R^{2}(\xi, \alpha) d \xi=0
$$

Substituting (23) into Eq. (24) and solving for $\alpha$, we get

$$
\alpha=\frac{1}{6}\left(3 \gamma+\sqrt{3\left(2+3 \gamma^{2}+16 \beta\right)}\right)
$$

in which for $\beta=1$, the result of Ariel [13] can be recovered. Thus, the original function $f$ now takes the form

$$
f=\gamma+\frac{1}{\alpha}(\exp (-\alpha \eta)+\alpha \eta-1)
$$


It is of interest to note that Eq. (26) is the solution for system (5) and (6) for all $\beta$ and $\gamma$.

For impermeable surface $\gamma=0$, Afzal and Luthra [15] have given the value $f^{\prime \prime}(0)$ for different values of $\beta$ in terms of higher order perturbations. The prediction of skin friction for large $\beta$, the asymptotic solution is given by

$$
f^{\prime \prime}(0)=\beta^{\frac{1}{2}}\left(1.1547+0.0746 \beta^{-1}+0.00509 \beta^{-2}-0.00182 \beta^{-3}+\cdots\right)
$$

and accelerated series in Euler transformation is given by

$$
f^{\prime \prime}(0)=Z^{-\frac{1}{2}}\left(1.1547-0.50272 Z-0.01019 Z^{2}-0.0384 Z^{3}+\cdots\right)
$$

where $Z=(1+\beta)^{-1}$. The results of this section are given in Tables 2 and 3 for different values of $\beta$ and $\gamma$ and also the results for $\gamma=0$ are given in Table 4 .

Table 3

\begin{tabular}{|c|c|c|c|}
\hline$\eta$ & $\begin{array}{l}\text { Eqs. (7) and (12) } \\
f^{\prime}(\eta)\end{array}$ & $\begin{array}{l}\text { Eq. }(26) \\
f^{\prime}(\eta)\end{array}$ & $\begin{array}{l}\text { Numerical solution } \\
f^{\prime}(\eta)\end{array}$ \\
\hline 0.0 & 0.0000000 & 0.0000000 & 0.0000000 \\
\hline 0.125 & 0.3730199 & 0.358335 & 0.3729810 \\
\hline 0.250 & 0.6157238 & 0.588266 & 0.6156581 \\
\hline 0.375 & 0.7686961 & 0.735805 & 0.7686340 \\
\hline 0.500 & 0.8628742 & 0.830475 & 0.8628434 \\
\hline 0.625 & 0.9197948 & 0.891222 & 0.9198138 \\
\hline 0.750 & 0.9536687 & 0.930201 & 0.9537486 \\
\hline 0.875 & 0.9735521 & 0.955212 & 0.9737001 \\
\hline 1.000 & 0.9850750 & 0.971261 & 0.9852978 \\
\hline 1.125 & 0.9916711 & 0.981559 & 0.9919770 \\
\hline 1.250 & 0.9954014 & 0.988167 & 0.9958015 \\
\hline 1.375 & 0.9974851 & 0.992407 & 0.9979946 \\
\hline 1.500 & 0.9986342 & 0.995128 & 0.9992723 \\
\hline 1.625 & 0.9992591 & 0.996874 & 1.0000499 \\
\hline 1.750 & 0.9995931 & 0.997994 & 1.0005660 \\
\hline 1.875 & 0.9997674 & 0.998713 & 1.0009577 \\
\hline 2.000 & 0.9998547 & 0.999174 & 1.0013042 \\
\hline 2.125 & 0.9998947 & 0.999470 & 1.0016527 \\
\hline 2.250 & 0.9999088 & 0.999660 & 1.0020328 \\
\hline 2.375 & 1.0000845 & 0.999782 & 1.0024655 \\
\hline 2.500 & 1.0001547 & 0.999860 & 1.0029672 \\
\hline 2.625 & 1.0002421 & 0.999910 & 1.0035529 \\
\hline 2.750 & 1.0003509 & 0.999942 & 1.0042377 \\
\hline 2.875 & 1.0004841 & 0.999963 & 1.0050375 \\
\hline 3.000 & 1.0006440 & 0.999976 & 1.0059699 \\
\hline 3.125 & 1.0008322 & 0.999985 & 1.0070541 \\
\hline 3.250 & 1.0011496 & 0.999990 & 1.0083120 \\
\hline 3.375 & 1.0012969 & 0.999994 & 1.0097677 \\
\hline 3.500 & 1.0015741 & 0.999996 & 1.0011448 \\
\hline 3.625 & 1.0018810 & 0.999997 & 1.0013384 \\
\hline 3.750 & 1.0022170 & 0.999998 & 1.0015609 \\
\hline 3.875 & 1.0025814 & 0.999999 & 1.0018161 \\
\hline 4.000 & 1.0029731 & 0.999999 & 1.0021081 \\
\hline
\end{tabular}

Comparison of solutions (7) and (12) and solution Eq. (26) with numerical solution of the Falkner-Skan equation (5), (6) with $\beta=4.0$ and $\gamma=2.0$.

Table 4

Comparison of value $f^{\prime}(0)$ for general $\beta$ and for impermeable surface $\gamma=0$.

\begin{tabular}{lccc}
\hline$\beta$ & Method of stretching variable (26) & Series solution (28) & Numerical solution Brodie \& Banks [16] \\
\hline 0.5 & 0.9129 & 0.9343 & 0.9277 \\
1 & 1.2247 & 1.2347 & 1.2326 \\
2 & 1.6833 & 1.6876 & 1.6872 \\
2.5 & 2.6141 & 1.8743 & 2.6158 \\
10 & 3.6742 & 3.6752 & 3.6752 \\
20 & 5.1801 & 5.1807 & 5.1807 \\
50 & 8.1752 & 8.1755 & 8.1755 \\
100 & 11.5546 & 11.5545 & 11.5545 \\
\hline
\end{tabular}




\section{Discussion and conclusions}

Representation of exact analytical solution (7) for $\beta=-1$ in the form of series expansion has encouraged us to analyze the similar behavior for other values of $\beta$. The exact solution (7) of the Falkner-Skan equation (5) subject to the boundary conditions (6) for general $\beta$ embeds the known analytical solution for $\beta=-1$ [8] and this solution holds for $\gamma \geqslant \sqrt{2}$. Whereas the solution (26) of the system (5) and (6) obtained through method of stretching of variables holds for general $\beta$ and $\gamma$. This method gives the best approximate solution for the Falkner-Skan equation and results are rather remarkable. This method is quite easy to use and requires less computational time. The solution (7) gives two-parameter family of solution of the problem (5). This should be matched with an analytical solution $\beta=-1$ to get one of the unknowns. Other unknown must be found from the integral relation (15) which itself is derived from system (5) and (6). We use the Pade' approximants to sum the series (12). With $\beta=0$, Eq. (5) reduces to the so-called Blasius equation, which describes a uniform boundary layer flow over semi-infinite plate with fluid saturated porous medium in the absence of pressure gradient and has been studied extensively and thus no comment is needed.

Solutions of the system (5) and (6) for velocity profile $f^{\prime}(\eta)$ against $\eta$ which are obtained from the relations (7) and (12) are displayed in Fig. 1a-d for different stretching parameter $\beta$ and suction parameter $\gamma$. The approach of the solution to its asymptotic value 1 (as $\eta \rightarrow \infty$ ) is clearly observed. For each $\beta$, there is gradual variation in velocity profile as $\gamma$ increases.

Tables 2 and 3 compare the solutions for $f^{\prime}(\eta)$ versus $\eta$ using (7) and (12) and from method of stretching of variables (i.e. Eq. (26)) for different values of $\beta$ with that of direct numerical solution of (5) and (6). There is an excellent agreement in all the three solutions. Comparison of the value $f^{\prime}(0)$ of the Falkner-Skan equations (5) and (6) obtained by method of stretching of variables with that of Eulerized series solution obtained by Afzal and Luthra [15] and numerically by Brodie and Banks [16] for different values of $\beta$, when $\gamma=0$ is given in Table 4. A close agreement between all solutions is clearly observed especially for larger values of $\beta$.

\section{Acknowledgements}

This work was supported by the UGC-Centre for Advanced Studies in Fluid Mechanics. Authors would like to thank the anonymous referee for useful critical comments.

\section{References}

[1] B.C. Sakiadis, Boundary layer behavior on continuous solid surfaces: the boundary layer on a continuous flat surface, A. I. Ch. E. J. 7 (1961) 221.

[2] N. Afzal, I.S. Varshney, The cooling of a low heat resistance stretching sheet moving through a fluid, Heat Mass Transfer 14 (1980) 289.

[3] N. Afzal, The cooling of a low heat resistance stretching sheet moving through a fluid: a rejoinder, Heat Mass Transfer 17 (1983) 217.

[4] N. Afzal, Heat transfer on stretching flat continuous surface, Int. J. Heat Mass Transfer 36 (1993) 1128.

[5] W.H.H. Banks, Similarity solutions of the boundary layer equations for a stretching wall, J. Mec. Theor. Appl. 2 (1983) 375.

[6] H. Weyl, On the differential equations of the simplest boundary layer problems, Ann. Math. 43 (1942) 381

[7] W.A. Coppel, On a differential equation of boundary layer theory, Phil. Trans. A 253 (1960) 101.

[8] H.T. Yang, L.C. Chien, Analytic solution of the Falkner-Skan equation when $\beta=-1$ and $\gamma=0$, SIAM J. Appl. Math. 29 (3) (1975) 558.

[9] N. Riley, P.D. Weidman, Multiple solutions of the Falkner-Skan equation for flow past a stretching boundary, SIAM J. Appl. Math. 49 (1989) 1350.

[10] N. Afzal, Momentum transfer on power law stretching plate with free stream pressure gradient, Int. J. Eng. Sci. 41 (2003) 1197.

[11] P.L. Sachdev, R.B. Kudenatti, N.M. Bujurke, Exact analytic solution of a boundary value problem for the Falkner-Skan equation, Stud. Appl. Math. 120 (2008) 1.

[12] N. Afzal, Minimum error solutions of boundary layer equations, Ind. Acad. Sci.: Math. Sci. Ser. A 91 (1982) 183.

[13] P.D. Ariel, Stagnation point flow with suction: an approximate solution, ASME Trans.: J. Appl. Mech. 61 (1994) 976-978.

[14] B.K. Chakraborthy, H.P. Mazumdar, MHD flow of a Newtonian fluid over a stretching sheet: an approximate solution, Approx. Theory Appl. 16 (2000) $32-41$.

[15] N. Afzal, V.K. Luthra, Highly accelerated laminar flow at moderately large Reynolds number, AIAA J. 12 (1974) 529-532.

[16] P. Brodie, W.H.H. Banks, Further properties of the Falkner-Skan equation, Acta Mech. 65 (1986) 205. 